\title{
Effect of multiple set on intramuscular metabolic stress during low-intensity resistance exercise with blood flow restriction
}

\author{
Tadashi Suga $\cdot$ Koichi Okita $\cdot$ Shingo Takada $\cdot$ Masashi Omokawa \\ Tomoyasu Kadoguchi · Takashi Yokota - Kagami Hirabayashi • \\ Masashige Takahashi $\cdot$ Noriteru Morita $\cdot$ Masahiro Horiuchi • \\ Shintaro Kinugawa $\cdot$ Hiroyuki Tsutsui
}

Received: 22 November 2011/ Accepted: 29 February 2012/Published online: 14 March 2012

(C) The Author(s) 2012. This article is published with open access at Springerlink.com

\begin{abstract}
Our previous study reported that intramuscular metabolic stress during low-intensity resistance exercise was significantly enhanced by combining blood flow restriction (BFR); however, they did not reach the levels achieved during high-intensity resistance exercise. That study was performed using a single set of exercise; however, usual resistance exercise consists of multiple sets with rest intervals. Therefore, we investigated the intramuscular metabolic stress during multiple-set BFR exercises, and compared the results with those during multiple-set highintensity resistance exercise. Twelve healthy young subjects performed 3 sets of 1-min unilateral plantar flexion (30 repetitions) with 1 -min intervals under 4 different conditions: low intensity (L, $20 \% 1 \mathrm{RM}$ ) and high intensity (H, $65 \% 1 \mathrm{RM})$ without $\mathrm{BFR}$, and $\mathrm{L}$ with intermittent
\end{abstract}

Communicated by Arnold de Haan.

T. Suga $\cdot$ S. Takada $\cdot$ T. Kadoguchi $\cdot$ T. Yokota $\cdot$

K. Hirabayashi $\cdot$ M. Takahashi - S. Kinugawa $\cdot$ H. Tsutsui

Department of Cardiovascular Medicine, Hokkaido University

Graduate School of Medicine, Sapporo, Japan

T. Suga

The Japan Society for the Promotion of Science, Tokyo, Japan

K. Okita $(\square)$

Graduate School of Program in Lifelong Learning Studies,

Hokusho University, 23 Bunkyodai, Ebetsu,

Hokkaido 069-8511, Japan

e-mail: okitak@hokusho-u.ac.jp

K. Okita $\cdot$ M. Omokawa $\cdot$ N. Morita $\cdot$ M. Horiuchi Northern Regions Lifelong Sports Research Center (SPOR), Ebetsu, Japan

N. Morita

Department of Sports Education, Hokkaido University

of Education, Iwamizawa, Japan
BFR (IBFR, only during exercise) and with continuous BFR (CBFR, during rest intervals as well as exercise). Intramuscular metabolic stress, defined as intramuscular metabolites and $\mathrm{pH}$, and muscle fiber recruitment were evaluated by ${ }^{31} \mathrm{P}$-magnetic resonance spectroscopy. The changes of intramuscular metabolites and $\mathrm{pH}$ during IBFR were significantly greater than those in L but significantly lower than those in H. By contrast, those changes in CBFR were similar to those in $\mathrm{H}$. Moreover, the fast-twitch fiber recruitment, evaluating by a splitting Pi peak, showed a similar level to $\mathrm{H}$. In conclusion, the multiple sets of lowintensity resistance exercise with continuous BFR could achieve with the same metabolic stress as multiple sets of high-intensity resistance exercise.

Keywords Energetic metabolism - Resistance training Magnetic resonance spectroscopy $\cdot$ Muscle hypertrophy

\section{Introduction}

Resistance training with high-intensity mechanical load can achieve muscle hypertrophy and strength increase; however, it generates intensive stress in musculo-skeletal and cardiovascular systems. Such high-intensity resistance training may induce orthopedic and cardiovascular problems. Thus, adequate high-intensity loads cannot be applied to elderly people and patients with various disorders (Williams et al. 2007). Despite using low-intensity mechanical loads, resistance training with blood flow restriction (BFR) has resulted in beneficial training effects (Clark et al. 2011; Karabulut et al. 2010; Takarada et al. 2002, 2004), which may be appropriate for physical fitness and rehabilitation in older people and those recovering from illness (Karabulut et al. 2010, 2011; Ohta et al. 2003; Ozaki et al. 2011). In previous 
studies, it has speculated that low-intensity resistance exercise with BFR might provide enhanced intramuscular metabolic stress and added skeletal muscle fiber recruitment (Fujita et al. 2007; Takarada et al. 2002, 2004); however, the exact mechanisms were not fully clarified.

In our previous studies (Suga et al. 2009, 2010), metabolic stress and muscle fiber recruitment during lowintensity resistance exercise were significantly increased with the addition of BFR; however, the effects of this combination did not reach the level of those during highintensity resistance exercise. The previous studies (Suga et al. 2009, 2010) used a single set of exercise; however, the usual resistance training involves multiple sets with rest intervals (Kraemer and Ratamess 2004). In fact, multipleset resistance training generally results in greater training effects than single-set (Krieger 2009, 2010).

The application of BFR during rest intervals in an exercise session is an important training variable, along with exercise intensity and BFR pressure (Cook et al. 2007). Continuous BFR during rest interval as well as exercise in multiple-set BFR exercise may be key factors for increasing metabolic stress and muscle fiber recruitment in skeletal muscle. Therefore, we evaluated the metabolic stress and fast-twitch (FT) fiber recruitment during multiple-set low-intensity BFR exercise, and then examined the effects of the BFR mode, either intermittent or continuous. Moreover, those BFR exercise protocols were compared to multiple-set highintensity resistance exercise.

\section{Methods}

\section{Subjects}

Twelve younger male subjects (age $22 \pm 4$ years, height $172 \pm 7 \mathrm{~cm}$, weight $69 \pm 7 \mathrm{~kg}$, mean $\pm \mathrm{SD}$ ) participated in the study. All subjects were healthy and without orthopedic or cardiovascular diseases. Subjects were tested on two occasions, separated by at least $48 \mathrm{~h}$. The subjects were instructed to refrain from transient strenuous physical activity and alcohol for at least $24 \mathrm{~h}$ prior to the testing. On the experiment day, subjects were required to abstain from caffeine and to fast for $4 \mathrm{~h}$ before the trial. The experimental trails were started from $\sim 1,800 \mathrm{~h}$ and then were arranged to be performed at the same time for each subject on the second experimental day. Informed consent was obtained from all subjects. This study was approved by the Ethics Committee of Hokusho University.

\section{Exercise protocols}

Subjects randomly performed the right ankle plantar flexion exercises with four different exercise conditions in two experimental days. Each experimental exercise session consisted of 3 sets of 1 min-exercise with 30 repetitions per set, lifting the weight $5 \mathrm{~cm}$ above ground. The rest intervals between sets were $1 \mathrm{~min}$ (Goto et al. 2005; Kraemer et al. 1990; Takarada et al. 2004). Thus, each experimental exercise session lasted $5 \mathrm{~min}$. Four exercise conditions were set as follows: two resistance exercises without BFR and two BFR exercise protocols. The two resistance exercises without BFR were low-intensity (L) and high-intensity $(\mathrm{H})$ exercises, at 20 and $65 \% 1 \mathrm{RM}$, respectively (Suga et al. 2009, 2010). The two BFR protocols were performed with intermittent or continuous BFR combined with L. In the intermittent BFR (IBFR) protocol, the BFR was applied only during exercise. The pressure cuff for IBFR was inflated before every set and released after the finish of sets. In the continuous BFR (CBFR) protocol, the BFR was maintained throughout the 5-min exercise session. The four exercise conditions were randomly performed on two exercise conditions per day. On each experimental day, the interval between conditions was at least $30 \mathrm{~min}$. Moreover, before the implementation of the second condition, the recovery in the subject's altered intramuscular metabolites and $\mathrm{pH}$ to baseline levels was confirmed. The subject's 1 RM was determined as successful contraction on the same plantar flexion apparatus equipped with a magnetic resonance device in the first experimental day. The $1 \mathrm{RM}$ trials were designed using increments of $10 \mathrm{~kg}$ until $60-80 \%$ of the perceived maximum. Then, the load was gradually increased by $1-5 \mathrm{~kg}$ weights until lift fails, in which the subject was not able to maintain proper form, or to completely lift the weight. The last acceptable lift with the highest possible load was determined as $1 \mathrm{RM}$. Mean $\pm \mathrm{SD}$ of resistance loads at 20 and $65 \% 1 \mathrm{RM}$ were $10 \pm 1$ and $33 \pm 4 \mathrm{~kg}$, respectively. BFR was carried out using a pneumatic rapid inflator (E-20 rapid cuff inflator, Hokanson, USA) with an 18.5-cm-wide pressure cuff placed around the right thigh. BFR pressure was applied $130 \%$ of the subject's resting systolic blood pressure (Clark et al. 2011; Cook et al. 2007; Takano et al. 2005; Suga et al. 2009, 2010) and $144 \pm 21 \mathrm{mmHg}$ on average.

\section{${ }^{31} \mathrm{P}$-magnetic resonance spectroscopy}

Subjects lay in the supine position on an original apparatus equipped with a magnetic resonance device, and the right foot was coupled to the pedal by a Velcro strap. ${ }^{31} \mathrm{P}-\mathrm{MRS}$ was performed using a $55-\mathrm{cm}$ bore, 1.5-tesla superconducting magnet (Magnetom Vision VB33G, Siemens Erlangen, Germany). An 80-mm surface coil was placed under the muscle belly of the right gastrocnemius. Shimming was adjusted using the proton signal from water. Spectra of high-energy phosphate 
were acquired at a pulse width of $500 \mu \mathrm{s}$, a transmitter voltage of $20 \mathrm{~V}$ and a repetition time of $2,000 \mathrm{~ms}$. The spectra were obtained at rest and during exercise. Each spectrum consisted of an average of 8 scans during $16 \mathrm{~s}$ before each time point. Peaks corresponding to highenergy phosphates were determined based on the chemical shifts. Peak areas were automatically calculated by peak fitting and integration after baseline correction using MR software. The phosphocreatine ( $\mathrm{PCr}$ ) millimolar concentration ([PCr]) assumed that $[\mathrm{PCr}]+$ creatine concentration $([\mathrm{Cr}])=42.5 \mathrm{mM}$ (Harris et al. 1974) and supposed that inorganic phosphate $\left(\mathrm{P}_{\mathrm{i}}\right)$ concentration $\left(\left[\mathrm{P}_{\mathrm{i}}\right]\right)$ is equal to $[\mathrm{Cr}]$ (Kemp and Radda 1994). The total change in $[\mathrm{PCr}]$ through the exercise sessions was quantified from the integrated area of the [PCr]-time curve. Diprotonated phosphate $\left(\mathrm{H}_{2} \mathrm{PO}_{4}{ }^{-}\right)$was calculated using $\left[\mathrm{P}_{\mathrm{i}}\right]$ (Lanza et al. 2005, 2007). Intramuscular $\mathrm{pH}$ was calculated from the chemical shift of Pi relative to PCr. When distinct Pi splitting was shown, the $\mathrm{pH}$ was calculated by standardizing the obtained individual $\mathrm{pH}$ on the basis of peaks corresponding to each Pi (Lanza et al. 2005, 2007).

\section{Statistical analyses}

Two-way ANOVA with repeated measures was used for comparisons among exercise conditions. Post hoc comparisons were made by Bonferroni's test. The comparisons of split-peak Pi appearance among exercise conditions were performed by a $\chi^{2}$ test. The level of significance was set at $P<0.05$. All statistical tests were performed using SPSS 13.0 for Windows software.

\section{Results}

At the end of the final sets in all four exercise conditions, intramuscular metabolites were significantly changed compared with resting values (respectively, $P<0.001$ ). Intramuscular $\mathrm{pH}$ at the end of the final set showed a significant decrease in two BFR protocols and $\mathrm{H}(P<0.001)$, but not in L, in comparison with that at rest. Changes of intramuscular metabolites and $\mathrm{pH}$ at the end of the final set in IBFR were significantly greater than those in L but significantly less than those in $\mathrm{H}$ (Table 1; Fig. 1). Those changes in CBFR were significantly greater than in IBFR $(P<0.001)$, and similar to $\mathrm{H}$ (Table 1; Fig. 1). The total change (the integrated area of the [PCr]-time curve) in $\mathrm{PCr}$ through each 5-min exercise session tended to be higher in IBFR than in $\mathrm{L}(P=0.074)$, but the difference did not reach statistical significance (Fig. 1). Total $\mathrm{PCr}$ change in CBFR was significantly greater than in IBFR $(P<0.001)$ and similar to $\mathrm{H}$ (Fig. 1).

The splitting of Pi peaks, representing the recruitment of FT fiber (Park et al. 1987; Vandenborne et al. 1991), was observed in two BFR protocols and $\mathrm{H}$, but not in $\mathrm{L}$. The number of subjects who showed split Pi peak during the final set in CBFR was significantly higher than that in L and IBFR $(P<0.001)$ and similar to that in $\mathrm{H}($ Fig. 2$)$.

\section{Discussion}

The present study investigated the effect of multiple-set BFR exercise on intramuscular metabolic stress and skeletal muscle fiber recruitment, and then examined the

Table 1 Intramuscular metabolite concentrations and $\mathrm{pH}$ at rest and the end of final set

\begin{tabular}{|c|c|c|c|c|}
\hline & \multirow[t]{2}{*}{$\mathrm{L}$} & \multicolumn{2}{|l|}{ BFR protocols } & \multirow[t]{2}{*}{$\mathrm{H}$} \\
\hline & & IBFR & CBFR & \\
\hline \multicolumn{5}{|l|}{$\mathrm{Pi}, \mathrm{mM}$} \\
\hline Rest & $5.1 \pm 0.2$ & $4.6 \pm 0.2$ & $4.8 \pm 0.3$ & $4.5 \pm 0.3$ \\
\hline End of final set & $11.2 \pm 0.7^{*}$ & $17.5 \pm 1.3^{* \dagger}$ & $27.0 \pm 1.5^{* \dagger *}$ & $26.6 \pm 1.3^{*+*}$ \\
\hline \multicolumn{5}{|l|}{$\mathrm{H}_{2} \mathrm{PO}_{4}^{-}, \mathrm{mM}$} \\
\hline Rest & $1.8 \pm 0.3$ & $1.6 \pm 0.3$ & $1.7 \pm 0.4$ & $1.6 \pm 0.3$ \\
\hline End of final set & $3.9 \pm 0.9 *$ & $6.8 \pm 2.6^{* \dagger}$ & $12.2 \pm 3.0 * \dagger$ & $11.6 \pm 2.9^{* \dagger *}$ \\
\hline \multicolumn{5}{|l|}{ Intramuscular $\mathrm{pH}$} \\
\hline Rest & $7.01 \pm 0.01$ & $7.01 \pm 0.02$ & $7.01 \pm 0.02$ & $7.00 \pm 0.02$ \\
\hline End of final set & $7.03 \pm 0.02$ & $6.96 \pm 0.07 * \dagger$ & $6.84 \pm 0.07 *+*$ & $6.87 \pm 0.07 *+$ \\
\hline
\end{tabular}

Values are mean $\pm \mathrm{SD}$

$B F R$ blood flow restriction, $L$ low-intensity resistance exercise at $20 \% 1 \mathrm{RM}, H$ high-intensity resistance exercise at $65 \% 1 \mathrm{RM}, I B F R$ intermittent BFR exercise protocol, $C B F R$ continuous BFR exercise protocol

* Significant difference $(P<0.05)$ between rest and the end of final, ${ }^{\dagger}$ significant difference $(P<0.05)$ from $L$, ${ }^{*}$ significant difference $(P<0.05)$ from IBFR 
Fig. 1 Time course of phosphocreatine (PCr) concentration during exercise protocols Symbols indicate means and error bars indicate SE. Significant difference between condition; $* P<0.05$ versus $L,{ }^{\dagger} P<0.05$ versus $I B F R$
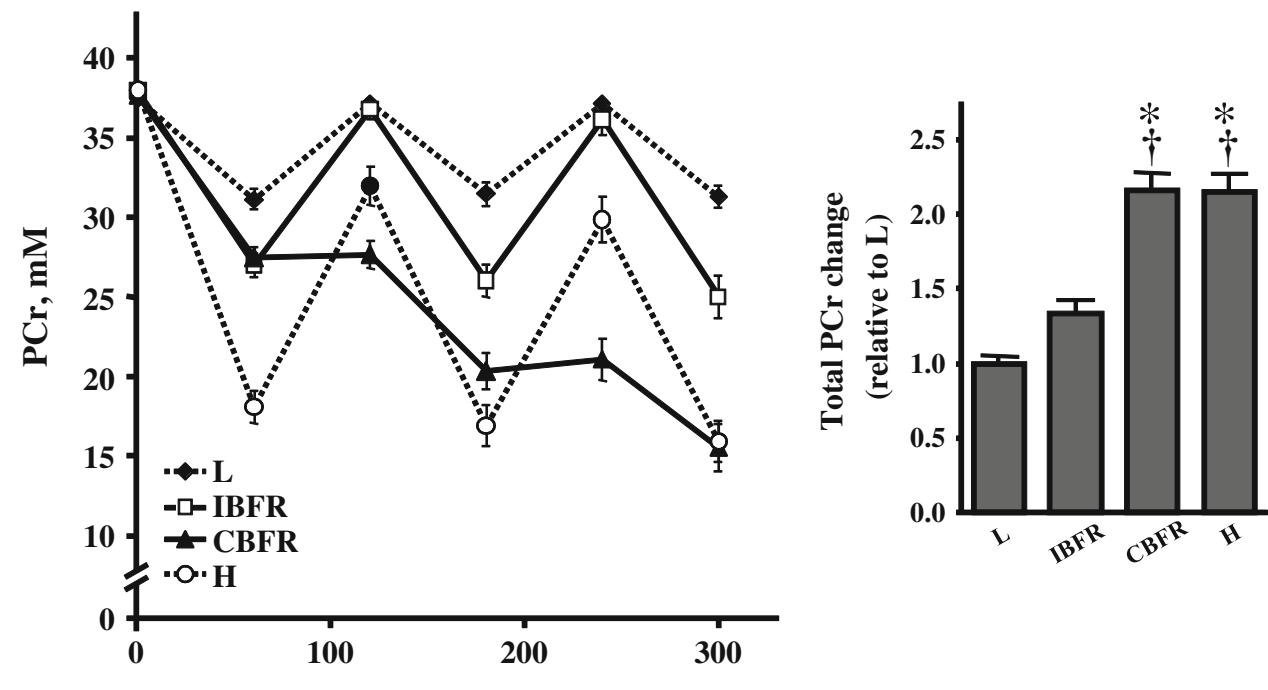

Time, sec

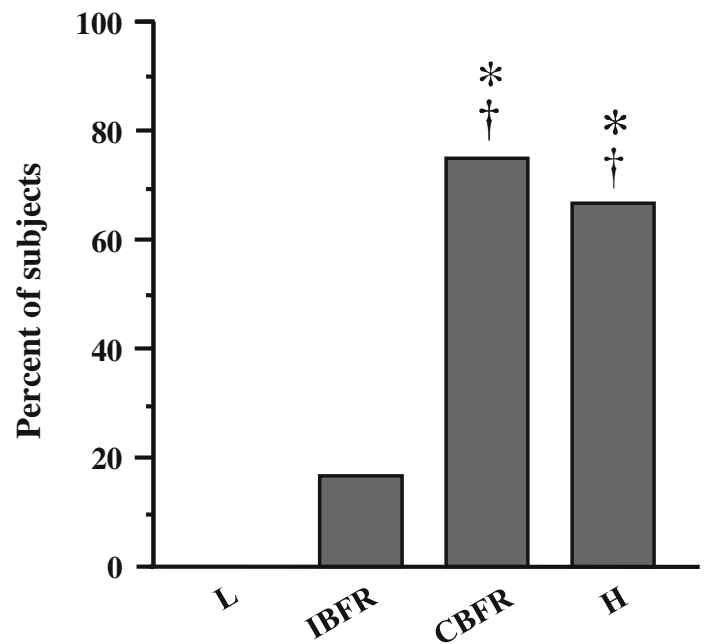

Fig. 2 The number of subjects who showed split-peak Pi. $* P<0.05$ versus $L,{ }^{\dagger} P<0.05$ versus $I B F R$

effects of the different BFR mode, intermittent and continuous. The results showed that metabolic stress, namely intramuscular metabolites and $\mathrm{pH}$, was significantly increased using intermittent restriction in the BFR protocol, but did not reach that of high-intensity resistance exercise. This result agrees with the result from a single set in our previous studies (Suga et al. 2009, 2010). However, the use of continuous restriction throughout the exercise session in the BFR protocol resulted in metabolic stress equivalent to that of high-intensity resistance exercise. The enhancement in FT fiber recruitment indicated as split Pi peak during exercise showed the same result. Thus, it was suggested that the multiple-set BFR exercise with continuous BFR might increase intramuscular metabolic stress and enhance skeletal muscle fiber recruitment equally to multiple-set high-intensity resistance exercise.
Harris and coworkers (Harris et al. 1976) found that the changes of intramuscular metabolites and $\mathrm{pH}$ after exercise are maintained by applying ischemic cuff pressure; this is called a "metabolic freeze" (Okita et al. 1998). Metabolic freeze method might be a useful tool to enhance training effect in BFR training. In the result of our present study, the changes in intramuscular metabolites and $\mathrm{pH}$ induced by exercise could be retained by continuing BFR during rest intervals. Such a technique might play an important role in promoting metabolic stress during BFR exercise. Accumulating evidence suggests that high-metabolic accumulation during resistance exercise can strongly stimulate growth hormone $(\mathrm{GH})$ release and skeletal muscle growth (Goto et al. 2005; Kraemer et al. 1990; Takarada et al. 2002). For example, Kraemer et al. (1990) and other studies (Takarada et al. 2002) have shown that a resistance exercise protocol with short rest intervals could effectively enhance the post-GH response and muscular training adaptations. This method of resistance training has something in common with the continuous BFR protocol in terms of inhibiting metabolic recovery during rest intervals. Thus, we propose that the manipulation of rest intervals in resistance exercise might play a key role for obtaining successful training effects.

In our previous study (Suga et al. 2009), splitting of Pi peaks during a single set of BFR protocol using the same intensity and pressure as that in the present study was observed in only $31 \%$ of subjects. This percent was significantly lower than that $(70 \%)$ during high-intensity resistance exercise. By contrast, the present findings showed that the number of subjects showing split-peak Pi during multi-set BFR protocol with continuous BFR was progressively increased as the number of sets, reaching a rate of $75 \%$ of subjects in the final set of exercise, despite the use of the same typical exercise intensity and BFR 
pressure as in our previous study (Suga et al. 2009). Moreover, this incidence was the same as that in multipleset high-intensity resistance exercise. Thus, by applying continuous restriction, multiple-set BFR exercise not only increases metabolic stress but also enhances FT fiber recruitment equal to that in high-intensity resistance exercise. According to Henneman's size principle, slow twitch (ST) fibers with small motor units are predominantly recruited during low-level muscle contraction (Henneman et al. 1965). Despite using low-intensity load, however, BFR exercise could recruit FT fibers to assist the ST fibers because of the maintenance of muscular force during exercise. The additional FT fiber recruitment during BFR exercise may be involved in the decreased oxygen supply and increased metabolic accumulation in working muscle. It is known that resistance training-induced muscular adaptation occurs more extensively in FT fiber than in ST fiber (Charette et al. 1991; McCall et al. 1996), suggesting that FT fiber recruitment during resistance exercise is required to obtain a significant training effect. Therefore, the results of the present study suggest that BFR during low-intensity resistance exercise might be a more effective supplement in multiple set than that in single-set protocols.

As one of its limitations, the present study recruited only healthy young subjects. The majority of previous BFR studies have also examined the training effects on healthy young adult subjects (Clark et al. 2011; Cook et al. 2007; Fujita et al. 2007; Takano et al. 2005, Takarada et al. 2002, 2004). Recent studies (Karabulut et al. 2010, 2011; Ozaki et al. 2011) have examined the effects of BFR exercise in older subjects, and have determined that BFR exercise could effectively lead to muscle hypertrophy and muscle strength increase. Karabulut et al. (2010) reported that the increase of muscle strength induced by BFR exercise was as effective as that of high-intensity resistance training. Moreover, Lanza et al. (2007) reported that the change of intramuscular metabolites during exercise under ischemia was similar in young and older subjects; however, the exercise modality in their study was maximal isometric contraction. Therefore, further study would be needed to determine the metabolic responses during BFR exercise in older subjects or patients with diseases. On the other hand, a previous study (Karabulut et al. 2011) reported that BFR resistance exercise could improve bone marker in blood level equally to high-intensity resistance exercise despite using low-intensity mechanical load. It suggests that BFR resistance exercise is also a potentiality effective training method to bone in addition to muscle. Thus, BFR resistance exercise may be most beneficial for weak individuals such as older people and diseased patients, including chronic heart failure, chronic obstructive pulmonary disease, cancer, and osteoporosis.

In conclusion, the present study showed that multiple-set low-intensity BFR exercise with continuous BFR could achieve intramuscular metabolic stress and skeletal muscle fiber recruitment equal to those of high-intensity resistance exercise.

Acknowledgments The authors thank Takashi Sato for assistance with data collection. Most notably, we are grateful to all subjects who gave of their time and effort to participate in this study. This work was supported by grants from Hokusho University Northern Regions Lifelong Sports Research Center (SPOR), Meiji Yasuda Life Foundation of Health and Welfare, and The Descente and Ishimoto Memorial Foundation for The Promotion of Sports Science.

Open Access This article is distributed under the terms of the Creative Commons Attribution License which permits any use, distribution, and reproduction in any medium, provided the original author(s) and the source are credited.

\section{References}

Charette SL, McEvoy L, Pyka G, Snow-Harter C, Guido D, Wiswell RA, Marcus R (1991) Muscle hypertrophy response to resistance training in older women. J Appl Physiol 70:1912-1916

Clark BC, Manini TM, Hoffman RL, Williams PS, Guiler MK, Knutson MJ, McGLynn ML, Kushnick MR (2011) Relative safety of 4 weeks of blood flow-restricted resistance exercise in young, healthy adults. Scand J Med Sci Sports 21:653-662

Cook SB, Clark BC, Ploutz-Snyder LL (2007) Effects of exercise load and blood-flow restriction on skeletal muscle function. Med Sci Sports Exerc 39:1708-1713

Fujita S, Abe T, Drummond MJ, Cadenas JC, Dreyer HC, Sato Y, Volpi E, Rasmussen BB (2007) Blood flow restriction during low-intensity resistance exercise increase S6K1 phosphorylation and muscle protein synthesis. J Appl Physiol 103:903-910

Goto K, Ishii N, Kizuka T, Takamatsu K (2005) The impact of metabolic stress on hormonal responses and muscular adaptations. Med Sci Sports Exerc 37:955-963

Harris RC, Hultman E, Nordesjö LO (1974) Glycogen, glycolytic intermediates and high-energy phosphates determined in biopsy samples of musculus quadriceps femoris of man at rest. Method and variance of values. Scand J Clin Lab Invest 33:109-120

Harris RC, Edwards RH, Hultman E, Nordesjö LO, Nylind B, Sahlin K (1976) The time course of phosphorylcreatine resynthesis during recovery of the quadriceps muscle in man. Pflugers Arch 367:137-142

Henneman E, Somjen G, Carpenter DO (1965) Functional significance of cell size in spinal motoneurons. J Neurophysiol 28:865879

Karabulut M, Abe T, Sato Y, Bemben MG (2010) The effects of lowintensity resistance training with vascular restriction on leg muscle strength in older men. Eur J Appl Physiol 108:147-155

Karabulut M, Bemben DA, Sherk VD, Anderson MA, Abe T, Bemben MG (2011) Effects of high-intensity resistance training and low-intensity resistance training with vascular restriction on bone markers in older men. Eur J Appl Physiol 111:1659-1667

Kemp GJ, Radda GK (1994) Quantitative interpretation of bioenergetic data from ${ }^{31} \mathrm{P}$ and ${ }^{1} \mathrm{H}$ magnetic resonance spectroscopic studies of skeletal muscle: an analytical review. Magn Reson Q 10:43-63

Kraemer WJ, Ratamess NA (2004) Fundamentals of resistance training: progression and exercise prescription. Med Sci Sports Exerc 36:674-688

Kraemer WJ, Marchitelli L, Gordon SE, Harman E, Dziados JE, Mello R, Frykman P, McCully D, Fleck SJ (1990) Hormonal and 
growth factor responses to heavy resistance exercise protocol. J Appl Physiol 69:1442-1450

Krieger JW (2009) Single versus multiple sets of resistance exercise: a meta-regression. J Strength Cond Res 23:1890-1901

Krieger JW (2010) Single versus multiple sets of resistance exercise for muscle hypertrophy: a meta-analysis. J Strength Cond Res 24:1150-1159

Lanza IR, Befroy DE, Kent-Braun JA (2005) Age-related changes in ATP-producing pathway in human skeletal muscle in vivo. J Appl Physiol 99:1736-1744

Lanza IR, Larsen RG, Kent-Braun JA (2007) Effects of old age on human skeletal muscle energetics during fatiguing contractions with and without blood flow. J Physiol 583:1093-1105

McCall GE, Byrnes WC, Dickinson A, Pattany PM, Fleck SJ (1996) Muscle fiber hypertrophy, hyperplasia, and capillary density in college men after resistance training. J Appl Physiol 81:2004-2012

Ohta H, Kurosawa H, Ikeda H, Iwase Y, Satou N, Nakamura S (2003) Low-load resistance muscular training with moderate restriction of blood flow after anterior cruciate ligament reconstruction. Acta Orthop Scand 74:62-68

Okita K, Yonezawa K, Nishijima H, Hanada A, Ohtsubo M, Kohya T, Murakami T, Kitabatake A (1998) Skeletal muscle metabolism limits exercise capacity in patients with chronic heart failure. Circulation 98:1886-1891

Ozaki H, Sakamaki M, Yasuda T, Fujita S, Ogasawara R, Sugaya M, Nakajima T, Abe T (2011) Increases in thigh muscle volume and strength by walk training with leg blood flow reduction in older participants. J Gerontol A Biol Sci Med Sci 66:257-263

Park JH, Brown RL, Park CR, McCully K, Cohn M, Haselgrove J, Chance B (1987) Functional pools of oxidative and glycolytic fibers in human muscle observed by ${ }^{31} \mathrm{P}$ magnetic resonance spectroscopy during exercise. Proc Natl Acad Sci USA 84:89768980

Suga T, Okita K, Morita N, Yokota T, Hirabayashi K, Horiuchi M, Takada S, Takahashi T, Omokawa M, Kinugawa S, Tsutsui H
(2009) Intramuscular metabolism during low-intensity resistance exercise with blood flow restriction. J Appl Physiol 106:11191124

Suga T, Okita K, Morita N, Yokota T, Hirabayashi K, Horiuchi M, Takada S, Omokawa M, Kinugawa S, Tsutsui H (2010) Doseeffect on intramuscular metabolic stress during low-intensity resistance exercise with blood flow restriction. J Appl Physiol 108:1563-1567

Takano H, Morita T, Iida H, Asada KI, Kato M, Uno K, Hirose K, Matsumoto A, Takenaka K, Hirata Y, Eto F, Nagai R, Sato Y, Nakajima T (2005) Hemodynamic and hormonal responses to a short-term low-intensity resistance exercise with the reduction of muscle blood flow. Eur J Appl Physiol 95:65-73

Takarada Y, Ishii N (2002) Effects of low-intensity resistance exercise with short interest rest period on muscular function in middle-aged women. J Strength Cond Res 16:123-128

Takarada Y, Sato Y, Ishii N (2002) Effects of resistance exercise combined with blood flow restriction on muscle function in athletes. Eur J Appl Physiol 86:308-314

Takarada Y, Tsuruta T, Ishii N (2004) Cooperative effects of exercise and occlusion stimuli on muscular function in low-intensity resistance exercise with moderate blood flow restriction. Jpn J Physiol 54:585-592

Vandenborne K, McCully K, Kakihira H, Parammer M, Bolinger L, Detre JA, De Meirlier K, Walter G, Chance B, Leigh JS (1991) Metabolic heterogeneity in human calf muscle during maximal exercise. Proc Natl Acad Sci USA 88:5714-5718

Williams MA, Haskell WL, Ades PA, Amsterdam EA, Bittner V, Franklin BA, Gulanick M, Laing ST, Stewart KJ (2007) Resistance exercise in individuals with and without cardiovascular disease: 2007 update-a scientific statement from the American Heart Association Council on Clinical Cardiology and Council on Nutrition, Physical Activity, and Metabolism. Circulation 116:572-584 